\title{
Nurturing instead of depleting empathy during medical education: a medical student's perspective
}

\section{Simone Chiang}

Faculty of Medicine, University of British Columbia, Vancouver, Canada

With the shift towards patient-centered care, it becomes increasingly evident that good medicine does not simply require technical skills in physical healing; a great physician also requires skills in communication, collaboration, leadership and compassion. Empathy is arguably at the core of these skills. While the exact definition is debated, empathy generally refers to the sharing of another person's experience or perspective and communicating this understanding [1].

Discouragingly, multiple studies report that empathy levels decline as medical students, residents, and physicians continue on in their education and careers [2]. While I am only a first year medical student, I already have a sense of this trend and can speculate three main reasons why empathy depletion occurs.

From my observations, first, there is the issue of educational stress and pressures. With the amount of medical knowledge expanding at a faster rate than ever and with increasing expectations asked of us in our academic and extra-curricular lives, students can feel overwhelmed. It can feel as if there is simply no room to focus on the emotional aspects of medical practice. Personally, I appreciate the efforts my medical school, the University of British Columbia (UBC), has taken to decrease this stress load by implementing the pass or fail system and allowing space for individualized styles of learning. With decreased pressure of academic competition and confinement, I feel there is more room for emotional development both personally and professionally.

Second, there is a widespread belief that if you are too compassionate and caring, then you will experience emotional burnout. This idea predisposes students to losing empathy proactively. In first year alone, my class has received multiple sessions on the trials of "emotional resilience' and I have had similar discussions with mentors. While I recognize and appreciate my school's efforts to give us realistic expectations for future obstacles, hearing of the seemingly inevitable emotional suffering and trauma we may experience as physicians if we are too emotionally invested can be very distressing. Students can feel discouraged from experiencing em-
Received: July 27, 2018 • Accepted: September 11, 2018

Corresponding Author: Simone Chiang (https://orcid.org/0000-0002-8041-4443)

Faculty of Medicine, University of British Columbia, 2329 West Mall, Vancouver, BC V6T 1Z4,

Canada

email: simone.chiang@alumni.ubc.ca
Korean J Med Educ 2018 Dec; 30(4): 365-368.

https://doi.org/10.3946/kjme.2018.111

eISSN: 2005-7288

(C) The Korean Society of Medical Education. All rights reserved. This is an open-access article distributed under the terms of the Creative Commons Attribution Non-Commercial License (http:// creativecommons.org/licenses/by-nc/3.0/), which permits unrestricted non-commercial use, distribution, and reproduction in any medium, provided the original work is properly cited. 
pathy and may put unnecessarily high guards up in acts of self-preservation before they are even exposed to cases of patient suffering.

In the current culture of empathy education, the impression is that empathetic behavior has many benefits for patients but only holds risks for a physician's well-being. During my research of empathy education in medical students at Korea University I was surprised to find many studies reporting that higher levels of empathy are actually protective for physicians: physicians with higher levels of empathy have been shown to have lower rates of burnout [3], a higher sense of well being [4], and decreased incidents of medical liabilities [5]. Teaching students to build appropriate professional and emotional barriers is undoubtedly important, but it would be more helpful if faculty and mentors balanced their approach in warning students with the benefits and strengths of empathy as well.

Finally, the third reason for empathy depletion may lie in the current format of empathy education and assessment. Recently, medical schools have been placing a greater emphasis on teaching and assessing empathy [6]. My university teaches students to express empathy by eliciting the patient's feelings, ideas, how the illness is affecting their functioning in life, and expectations during patient interviews. Many similar templates exist but with any of these approaches come empathetic statements such as: "This is clearly troubling for you. How does this make you feel?' We are taught that the wording is crucial and to be very particular in the phrasing. Students practice these lines intensively with standardized patients and then use them in our biannual objective structured clinical examination (OSCE). As a result of rehearsing in artificial settings and feeling pressured to use specific vocabulary off of the invigilator's checklist, students can sound automatic and insincere. But real world scenarios involve real patients with real feelings and concerns, and no invigilator is assessing us with a checklist in mind. Students must be reminded that without empathy we not only fail our OSCE, but more importantly, we fail our patients in real practice.

Arguably, empathy training and specific phrases do improve patient rapport and outcomes as shown in a randomized controlled trial [7]. If the empathy is perceived as genuine by patients, then it may not seem as important whether physicians truly feel empathetic or if they are merely acting. However, as mentioned earlier, there are many benefits for a physician's internal sense of empathy and therefore developing genuine empathy is in the best interest for both the patient and physician.

As a student, I understand that certain aspects of empathy depletion are out of the faculty's control such as inappropriate informal empathy teaching (e.g., inadequate mentors, unsuitable learning environments) or the hidden medical curricula (e.g., student mistreatment) [8]. I also recognize that medical schools are teaching institutes; they do not exist to nurture each student into upstanding adults as a parent would. However, I do believe that to best cultivate empathy, empathetic abilities must be nurtured rather than simply taught. While teaching simply implies transference of knowledge or skills, nurturing involves creating an environment that cultivates attitudes, thoughts, and actions reflecting an innate quality such as empathy. Since studies show that students and physicians lose empathy throughout time, there must exist a certain level of empathy in each student at the start of their medical education. Instead of depleting these levels, medical schools can better preserve and nurture their students' empathy through various feasible methods.

First-hand experiences and work-based learning are key strategies to grow empathy in students. Exposing students to clinical experiences early on in their edu- 
cation is very impactful. At UBC, first year students are placed into community-based family practice clinics one day a week for half of each semester. We also receive other opportunities for clinical placements through shadowing community physicians, or in our Flexible Enhanced Learning course (FLEX), where students are allowed to pursue individualized learning experiences. During my FLEX, I was able to work in a Hepatitis C Community Clinic serving predominantly intravenous drug users and a homeless population. Undoubtedly, at the end of my 6-week placement I was better able to connect and understand this patient population after witnessing the socio-economic barriers and discrimination so many of them face on a daily basis.

Through work-based learning, many of my peers and I have been able to gain a more holistic view of patients and understand what symptoms, side effects, and socio-economic barriers truly look like on a person, which didactic lectures could never convey. Especially when we are placed long enough to see the same patient on multiple visits, students can develop relationshipbuilding skills that are simply impossible via standardized patients and workshops. A key component of empathy is the sharing of a person's experience; therefore, early first-hand exposure to patient experiences seems like an obvious and natural way to nurture empathy in medical students.

Interprofessional education (IPE) also holds great potential to nurture empathy. The use of IPE sessions not only allows for students to practice interdisciplinary teamwork and communication, but also help medical students gain a richer understanding of other healthcare professions. With this understanding comes a deeper view of what patients are experiencing in the health care system and in their life, which can never be achieved from a solely medical aspect. For example, as a medical student, I never considered how exactly a patient's home must be modified after a spinal injury, as an occupational therapist does. I also never considered the time, mental stress, and financial strain that can occur in trying to maintain a healthy diet for those with diabetes if they are living in low-income houses as a social worker or dietician do. While IPE sessions can seem troublesome and insignificant to students at first, if executed properly with enthusiastic engagement, IPE is a great exercise of sharing different perspectives and ultimately improving a student's ability to empathize with patients and other healthcare workers as well.

In summary, there seems to be a current resignation that medical students will lose their empathy and that there is a need teach them how to be empathetic. But with these aforementioned suggestions, perhaps we can move towards a culture and curriculum that nurtures the empathy and idealism medical students enter in with rather than re-teaching them a skill they've lost. Decreasing the academic load and pressure, eliminating the misconception that empathy only leads to burnout, and reminding students that empathy is not just an act to put on for OSCE purposes are all ways to inhibit empathy depletion. Furthermore, allowing for real patient interactions early on in the curriculum and holding well-executed interprofessional sessions can aid in nurturing empathy in medical students as well. Of course I recognize that changing an entire medical education environment seems radical and daunting; however, these suggested methods are very much attainable and when weighed against the benefits both the patients and our community of physicians may receive, I believe it is worth the cost and effort.

\section{ORCID:}

Simone Chiang: https://orcid.org/0000-0002-8041-4443

Acknowledgements: This essay was written during an 
exchange research program sponsored by the International Federation of Medical Students Assocation (IFMSA) at the Korea University College of Medicine, Seoul under the guidance of Dr. Young-Mee Lee.

Funding: None.

Conflicts of interest: No potential conflict of interest relevant to this article was reported.

Author contributions: Whole process, ranging from conception of the work to critical revision, was done by the author.

\section{References}

1. Hojat M, Gonnella JS, Mangione S, et al. Empathy in medical students as related to academic performance, clinical competence and gender. Med Educ. 2002;36(6): 522-527.

2. Neumann M, Edelhäuser F, Tauschel D, et al. Empathy decline and its reasons: a systematic review of studies with medical students and residents. Acad Med. 2011; 86(8):996-1009.

3. Von Harscher H, Desmarais N, Dollinger R, Grossman S,
Aldana S. The impact of empathy on burnout in medical students: new findings. Psychol Health Med. 2018;23(3): 295-303.

4. Shanafelt TD, West C, Zhao X, et al. Relationship between increased personal well-being and enhanced empathy among internal medicine residents. J Gen Intern Med. 2005;20(7):559-564.

5. Moore PJ, Adler NE, Robertson PA. Medical malpractice: the effect of doctor-patient relations on medical patient perceptions and malpractice intentions. West J Med. 2000; 173(4):244-250.

6. Richards N, Coulter A; Picker Institute Europe. Is the NHS becoming more patient-centred?: trends from the national surveys of NHS patients in England 2002-07. Oxford, UK: Picker Institute Europe; 2007.

7. Wündrich M, Schwartz C, Feige B, Lemper D, Nissen C, Voderholzer U. Empathy training in medical students: a randomized controlled trial. Med Teach. 2017;39(10): 1096-1098.

8. Kelm Z, Womer J, Walter JK, Feudtner C. Interventions to cultivate physician empathy: a systematic review. BMC Med Educ. 2014;14:219. 